\title{
Unusual Presentation of Atypical Kawasaki Disease in a Child with Thrombocytopenia and Spontaneous Femoral Artery Leak: A Case Report
}

\author{
Mohamed S. Eid ${ }^{1 *}$, Ahmed M. Badr' ${ }^{1}$, Hala S. Hamza', Yasser Sedky ${ }^{1}$ \\ ${ }^{1}$ Department of Pediatrics, Faculty of Medicine, Cairo University, Egypt; \\ mohamed_samir_eid@cu.edu.eg, abadr2007@yahoo.com,dr.hala_hamza@hotmail.com, \\ ymsedky@hotmail.com \\ * Correspondence: mohamed_samir_eid@cu.edu.eg. \\ Received: 9/3/2021; Accepted: 7/6/2021; Published online: 25/6/2021
}

\begin{abstract}
:
Kawasaki disease (KD) is an acute systemic vascular disease that affects mostly medium sized and small vessels. The most serious of which is coronary artery disease. Vasculitis involves proliferative granulomatous inflammation and resolves by cicatrization if not treated. It is generally a self-limited disease and its highest incidence is in children under five years. The diagnostic criteria include the presence of fever for at least 5 days along with four of five other clinical features (rash, mucositis, conjunctival injection, cervical lymphadenopathy or extremity changes). Atypical Kawasaki disease includes patients who meet only 2 or 3 of the 5 criteria for diagnosis. We here report a 4- year old male patient who presented with fever, mucositis, perineal rash and evidence of de novo femoral vasculitis leading to spontaneously leaking femoral arteritis. His complete blood count revealed thrombocytopenia. The patient fulfilled the criteria for the diagnosis of atypical Kawasaki Disease. He received intravenous immunoglobulins and aspirin in anti-inflammatory doses and his condition improved. We report this unusual presentation of Kawasaki disease with spontaneous femoral artery leak, rash and thrombocytopenia. Diagnosis of atypical Kawasaki disease is very challenging but essential as initiation of specific management is life-saving.
\end{abstract}

Level of Evidence of Study: IV (1).

Keywords: atypical Kawasaki disease; femoral artery aneurysm; thrombocytopenia.

Abbreviations: BCG: Bacillus Calmette-Guérin; KD: Kawasaki disease.

\section{Introduction}

Kawasaki disease (KD) is characterized as an acute systemic vascular disease that affects mostly medium sized and small vessels. It is generally a self-limited disease and its highest incidence in children is under five years(2). The diagnosis of $\mathrm{KD}$ relies mostly on clinical signs and symptoms. The diagnostic criteria require the presence of fever for at least 5 days along with four of five other clinical features (rash, mucositis, conjunctival injection, cervical lymphadenopathy or extremity changes). The American Heart Association suggests the use of supportive laboratory testing and echocardiography to help detect patients who are at risk (3). Atypical Kawasaki disease includes patients who meet only 2 or 3 of the 5 criteria for diagnosis (4). They can be missed by application of classic diagnostic criteria of The American Heart Association. The main complication of KD is coronary artery aneurysm (3). Suggested laboratory testing included markers of inflammation, presence of anemia, leukocytosis, thrombocytosis, hypoalbuminemia, elevated liver enzymes and possible sterile pyuria. An echocardiogram can detect presence of any early coronary artery abnormalities or involvement (5). BCG vaccine scar reactivation, perineal hyperemia, skin peeling and gall bladder hydrops are documented in many cases $(6,7)$. In developed countries, $\mathrm{KD}$ is the most common acquired heart disease in children and its most serious complication are coronary artery lesions, which have been reported in 20 $25 \%$ of untreated children (8). Medium sized vasculitis other than coronary artery in Kawasaki disease in children was reported to affect pulmonary artery, renal artery, portal vein, pancreatic vessels, intestinal subserosal arteries, testicular arteries, meningeal arteries and others (9). 
The purpose of this work was to report atypical presentation for Kawasaki disease and the course of management.

\section{Case Presentation}

A 4-year- old male child presented on the $12^{\text {th }}$ day of fever to our medical attention. Mother reported that child had a 10 days history of increasing irritability and poor feeding. On day 5 of fever, he developed a maculopapular rash on the trunk, chest and abdomen which disappeared on the next day. On day 7, he developed perianal hyperaemia and groin ecchymosis associated with red lips and tongue (Figure 1), for which he had some laboratory tests. There was no lymphadenopathy at any stage.
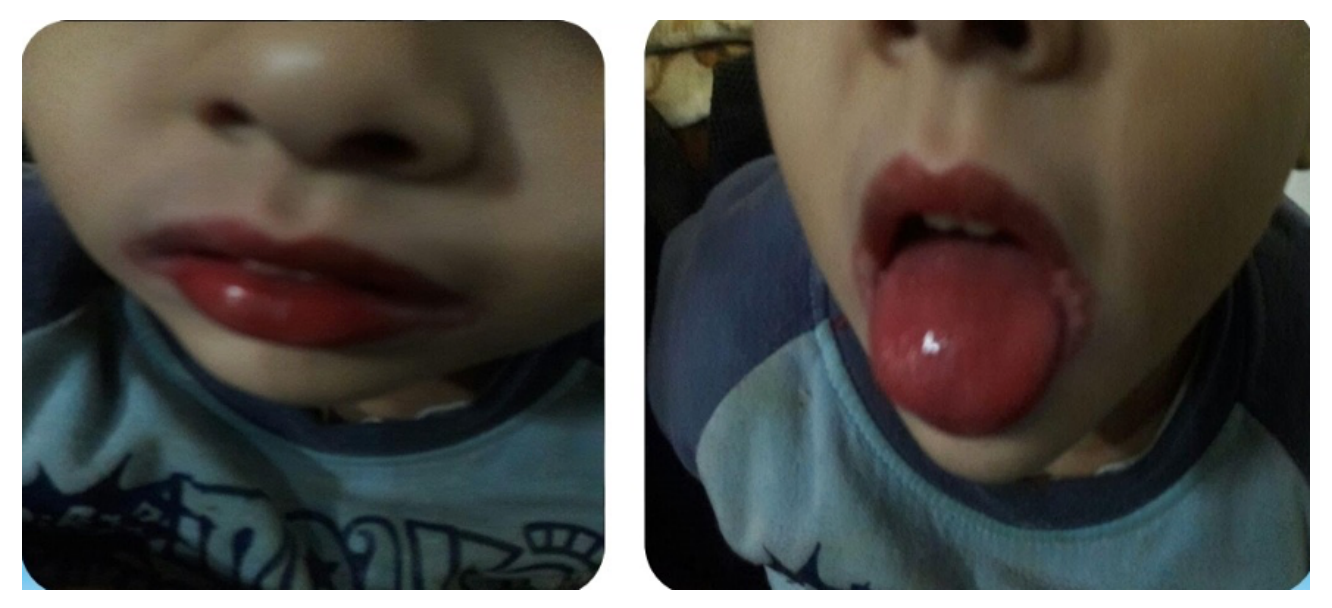

Figure (1): Oral mucositis in the studied child with atypical Kawasaki.

Initial labs revealed anaemia, thrombocytopenia, normal urine analysis and markedly elevated inflammatory markers. Blood culture and virology screening results were negative. Echocardiography revealed no abnormality.

On day 13 of fever, he developed perianal peeling (Figures 2 and 3), new labs revealed high inflammatory markers, sterile pyuria, mild elevation of alanine transaminase and hypoalbuminemia. Echocardiography was still normal. Groin ecchymosis was prominent. Bedside Doppler study revealed leaking femoral hematoma (pseudoaneurysm).
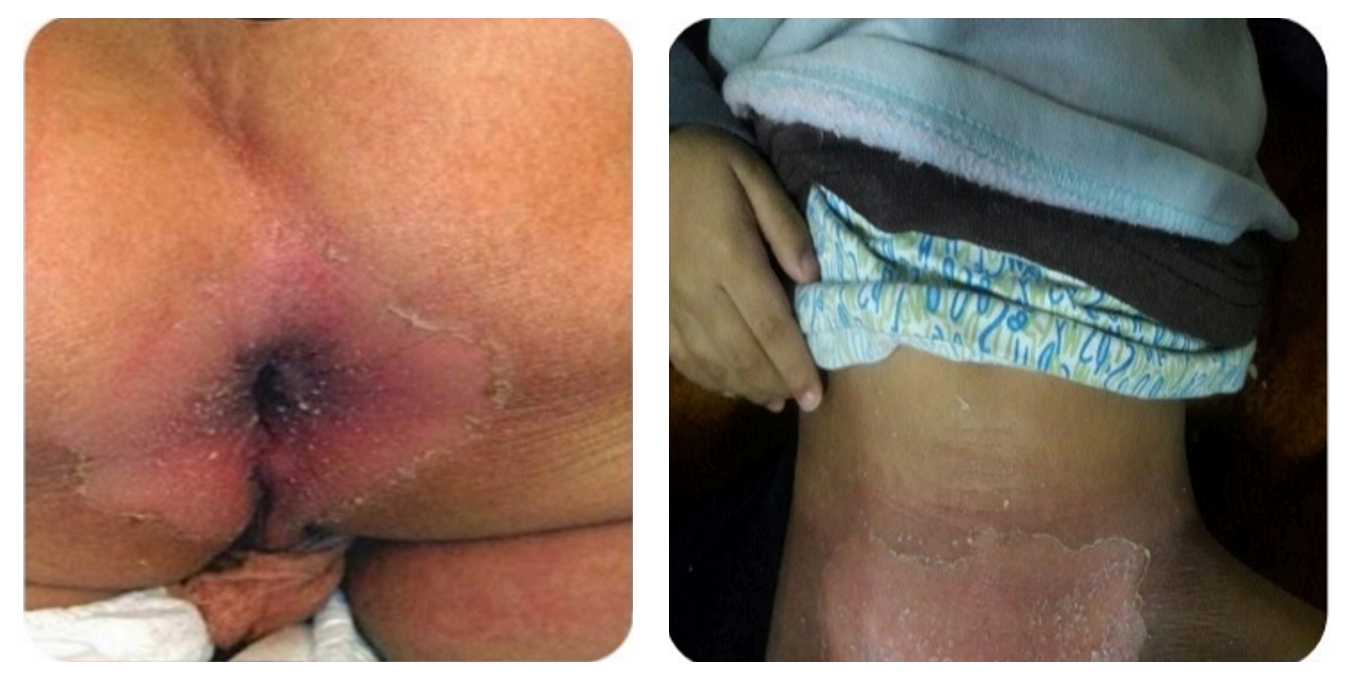

Figure (2): Perineal, groin and lower abdomen desquamation in the studied child with atypical Kawasaki.

At this stage on day 13, the criteria for atypical Kawasaki disease were fulfilled and intravenous immunoglobulins were initiated. Fever subsided and platelet count was the first to normalize. On day 14 of fever, anti-inflammatory dose of aspirin initiated. 
The general condition improved with normalization of previously elevated inflammatory markers and thrombocytosis started to appear in blood picture. Fortunately, echocardiography remained normal. Ecchymosis in the groins disappeared on follow up. Follow up Doppler demonstrated normal femoral arteries without evidence of aneurysmal dilatation. Table (1) summarizes laboratory results at day 7 and 13 of fever.

Table (I): Laboratory findings at day 7 and 13 of the studied child with atypical Kawasaki.

\begin{tabular}{lcc}
\hline & Day $\mathbf{7}$ & Day 13 \\
\hline Hb gm\% & 8.2 & 8.4 \\
\hline MCV & 66.8 & 68 \\
\hline TLC (in thousand) & 4.2 & 9.5 \\
\hline PLT (in thousand) & 51 & 313 \\
\hline CRP & 344 & 184 \\
\hline ESR (First hour) & 160 & 80 \\
\hline Urine & normal & Sterile Pyuria \\
\hline Blood c/s & No growth & Not repeated \\
\hline Virology & Negative & Not repeated \\
\hline ALT up to 40 U/L & 40 & 70 \\
\hline Albumin (gm\%) & 3.4 & 2.6 \\
\hline
\end{tabular}

CRP: C-reactive protein; ESR: erythrocyte sedimentation rate; Hb: hemoglobin; MCV: mean corpuscular volume; PLT: platelets; TLC: total leucocytic count; .

\section{Discussion}

Kawasaki disease is the second most common vasculitis of childhood, so it should be included in the differential diagnosis for any child with a prolonged unexplained fever (10).

While steroids are the first line of management in most of the immune vasculitis; its use is controversial in Kawasaki disease $(11,12)$. Intravenous immunoglobulins is the first line of management to prevent the coronary artery disease in Kawasaki disease (13). Incomplete or atypical Kawasaki disease should be taken into consideration in case of all children with unexplained fever for more than 5 days, associated with 2 or 3 of the main clinical findings of Kawasaki disease (4). The diagnosis of incomplete Kawasaki disease is based on presence of any three of the supplemental laboratory criteria: anaemia, leucocytosis, thrombocytosis, elevated alanine aminotransferase, hypoalbuminemia and sterile pyuria. Cardiac complications, mostly coronary artery aneurysm, can occur in $20 \%$ to $25 \%$ of untreated patients and in $4 \%$ of treated patients (13). Other aneurysms in different medium sized arteries are documented ( e.g. axillary and femoral arteries) (14).

As with our case, it is clear that we might miss cases with atypical Kawasaki due to the wide, unknown spectrum of Kawasaki. Hence, criteria of diagnosis of Kawasaki needs to be revised (15), to help more prompt diagnosis and initiation of therapy.

Groin ecchymosis was another challenging sign and was due to spontaneously leaking femoral artery. It is not clear however if the leak was due to the severe vasculitic process or due to the associated thrombocytopenia.

The resolution of Kawasaki disease and femoral artery injury in our studied child is contrary to fatal fate associated with ruptured aortic aneurysm (16). Diagnosis of atypical Kawasaki disease is very challenging but essential as initiation of specific management is life-saving.

\section{Conclusion}

Atypical Kawasaki disease spectrum includes thrombocytopenia, spontaneous-leaking femoral arteritis and evanescent rash. Spontaneous femoral artery leak in atypical Kawasaki disease responded to conservative management by intravenous immunoglobulins and antiinflammatory dose of aspirin. The variability in the clinical presentation and the sequential appearance of clinical and lab findings may delay the diagnosis. Therefore, the atypical picture of the disease should be kept in mind in order not to delay life-saving therapies. 
Author Contributions: All authors searched medical literature, databases, conceptualized, conducted the case review and reviewed the final manuscript. All authors have read and agreed to the published version of the manuscript.

\section{FUNDING}

Authors declare there was no extramural funding provided for this study.

\section{CONFLICT OF INTEREST}

The authors declare no conflict of interest. Authors declare veracity of information.

\section{References}

1. S. Tenny, M. Varacallo, Evidence Based Medicine. (StatPearls Publishing; Treasure Island (FL), 2020; https://www.ncbi.nlm.nih.gov/books/NBK470182/).

2. J. C. Burns, M. P. Glodé, Kawasaki syndrome. Lancet Lond. Engl. 364, 533-544 (2004).

3. H. S. Hamza, W. A. Raouf, A. Z. Zaher, H. M. Agha, Acute Kawasaki disease with emphasis on the echocardiographic profile: A single center experience. Glob. Cardiol. Sci. Pract. 2017, e201727 (2017).

4. S. K. Tanti, S. Mishra, Atypical Kawasaki Disease Complicated With Coronary Artery Aneurysm: A Case Report and Review of Literature. Cureus. 13, e13589 (2021).

5. J. W. Newburger, M. Takahashi, M. A. Gerber, M. H. Gewitz, L. Y. Tani, J. C. Burns, S. T. Shulman, A. F. Bolger, P. Ferrieri, R. S. Baltimore, W. R. Wilson, L. M. Baddour, M. E. Levison, T. J. Pallasch, D. A. Falace, K. A. Taubert, Committee on Rheumatic Fever, Endocarditis, and Kawasaki Disease, Council on Cardiovascular Disease in the Young, American Heart Association, Diagnosis, treatment, and long-term management of Kawasaki disease: a statement for health professionals from the Committee on Rheumatic Fever, Endocarditis, and Kawasaki Disease, Council on Cardiovascular Disease in the Young, American Heart Association. Pediatrics. 114, 1708-1733 (2004).

6. M. S. Rezai, S. Shahmohammadi, Erythema at BCG Inoculation Site in Kawasaki Disease Patients. Mater. Socio-Medica. 26, 256-260 (2014).

7. A. Gupta, S. Singh, Kawasaki disease for dermatologists. Indian Dermatol. Online J. 7, 461-470 (2016).

8. H.-C. Kuo, Preventing coronary artery lesions in Kawasaki disease. Biomed. J. 40, 141-146 (2017).

9. K. Takahashi, T. Oharaseki, Y. Yokouchi, N. Hiruta, S. Naoe, Kawasaki disease as a systemic vasculitis in childhood. Ann. Vasc. Dis. 3, 173-181 (2010).

10. A. Schnabel, C. M. Hedrich, Childhood Vasculitis. Front. Pediatr. 6, 421 (2019).

11. M. Levin, Steroids for Kawasaki disease: the devil is in the detail. Heart. 99, 69-70 (2013).

12. A. J. Wardle, H. C. Kiddy, M. J. Seager, R. M. Tulloh, M. Levin, in Cochrane Database of Systematic Reviews, The Cochrane Collaboration, Ed. (John Wiley \& Sons, Ltd, Chichester, UK, 2014; http://doi.wiley.com/10.1002/14651858.CD011188), p. CD011188.

13. M. S. Song, Predictors and management of intravenous immunoglobulin-resistant Kawasaki disease. Korean J. Pediatr. 62, 119-123 (2019).

14. N. Kumar, M. Mittal, M. Sinha, A. Gupta, B. Thukral, Unusual imaging presentation of infantile atypical Kawasaki disease. Indian J. Radiol. Imaging. 26, 373 (2016).

15. T. Kobayashi, M. Ayusawa, H. Suzuki, J. Abe, S. Ito, T. Kato, M. Kamada, J. Shiono, K. Suda, K. Tsuchiya, T. Nakamura, Y. Nakamura, Y. Nomura, H. Hamada, R. Fukazawa, K. Furuno, H. Matsuura, T. Matsubara, M. Miura, K. Takahashi, Revision of diagnostic guidelines for Kawasaki disease (6th revised edition). Pediatr. Int. 62, 1135-1138 (2020).

16. D. M. Hunsaker, J. C. Hunsaker, K. C. Adams, J. A. Noonan, D. M. Ackermann, Fatal Kawasaki disease due to coronary aneurysm rupture with massive cardiac tamponade. J. Ky. Med. Assoc. 101, 233-238 (2003).

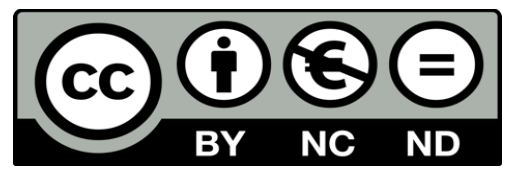

C 2021 submitted by the authors. Open access publication under the terms and conditions of the Creative Commons Attribution (CC- BYNC- ND) license. (https://creativecommons.org/licenses/by-nc$\mathrm{nd} / 2.0 /)$. 